\title{
Identificação e tratamento da dor no recém-nascido prematuro na Unidade de Terapia Intensiva
}

\author{
Identification and treatment of pain in the premature newborn in the Intensive Care Unit
} Identificación y tratamiento del dolor en el recién nacido prematuro en la Unidad de Terapia Intensiva

\section{Luciano Marques dos Santos', Isabelle Santos Ribeiro", Rosana Castelo Branco de Santana'II}

' Universidade Estadual de Feira de Santana, Curso de Graduação em Enfermagem,

Núcleo Interdisciplinar de Estudos das Desigualdades Sociais em Saúde. Feira de Santana-BA, Brasil.

"Fundação Hospitalar de Feira de Santana, Complexo Materno-Infantil, Setor de Educação Permanente. Feira de Santana-BA, Brasil. "' Universidade Estadual de Feira de Santana, Curso de Graduação em Enfermagem. (Graduanda) Feira de Santana-BA, Brasil.

\author{
Submissão: 21-01-2011 Aprovação: 13-06-2012
}

\section{RESUMO}

O estudo objetivou analisar os parâmetros utilizados pela equipe de enfermagem de um hospital público da Bahia para a avaliação da dor no recém-nascido prematuro e descrever as intervenções utilizadas para aliviar a dor. Estudo descritivo e qualitativo, realizado por meio de entrevistas semiestruturadas com dez participantes, no período de dezembro de 2008 a janeiro de 2009, sendo analisados através da Análise de Conteúdo. Os resultados apontaram a utilização do choro e expressão facial como parâmetros indicativos de dor; e que estes profissionais utilizam de forma não sistematizada medidas não farmacológicas para amenizar este processo. Sugere-se a introdução da dor como o quinto sinal vital a ser avaliado e a utilização de escalas, com vistas à excelência e humanização do cuidado.

Descritores: Enfermagem neonatal; Prematuro; Dor; Recém-nascido; Unidades de Terapia Intensiva Neonatal.

\begin{abstract}
This study aimed to analyze the parameters used by the nursing staff of a public hospital in Bahia for pain assessment in premature newborns and to describe the interventions used to relieve the pain. This is a qualitative descriptive study that was carried out through semi-structured interviews with ten participants, in the period from December 2008 to January 2009 . The data were analyzed through content analysis. The results showed the use of crying and facial expression as the clinical indications of pain premature newborns and that the interviewed participants use, on a non-systematic basis, non-pharmacological measures in order to ease this process. We suggest the introduction of pain as the fifth vital sign to be evaluated and the use of scales, contributing to excellence and humane care.

Key words: Neonatal nursing; Newborn, Premature; Pain; Newborn; Neonatal Intensive Therapy Unit.

\section{RESUMEN}

Este estudio tiene el objetivo de analizar los parámetros utilizados por el equipo de enfermeros de un hospital público de Bahía para la evaluación del dolor en el recién nacido prematuro y describir las intervenciones utilizadas para aliviar el dolor. Se trata de un estudio descriptivo y cualitativo, realizado a través de entrevistas semi estructuradas con diez participantes, en el período de diciembre de 2008 hasta enero de 2009, siendo analizados por medio del Análisis Contenido. Los resultados apuntaran la utilización del lloro y expresión facial como parámetros clínicos indicativos de dolor y que estos profesionales utilizan de manera no sistematizada medidas no farmacológicas con la finalidad de amenizar este proceso. Se sugiere a la introducción del dolor como el quinto señal vital a ser evaluado y la utilización de escalas, con el fin de la excelencia y humanización del cuidado.
\end{abstract}

Palabras clave: Enfermería neonatal; Prematuro; Dolor; Recién-nacido; Unidades de Terapia Intensiva Neonatal. 


\section{INTRODUÇÃO}

Nas últimas décadas a Neonatologia tem passado por profundas transformações, tanto do ponto de vista tecnológico, quanto da veiculação de evidências científicas que têm proporcionado melhorias significativas no cuidado ao Recém-Nascido Prematuro (RNPT) e sua família.

A sobrevida orgânica do RNPT tem melhorado, fazendo com que neonatos com idades gestacionais extremas e/ou de muito baixo peso ao nascimento sobrevivam. Apesar dos avanços tecnológicos e desenvolvimento do país, infelizmente as taxas de prematuridade permanecem elevadas. No Brasil, um país em desenvolvimento, a prevalência de prematuros é de aproximadamente $7 \%{ }^{(1)}$.

Observa-se um crescente aumento no número de RNPT que recebem alta das complexas Unidades de Terapia Intensiva Neonatal (UTIN). Este cenário pode decorrer das evidências científicas disponíveis para os trabalhadores da saúde, bem como da sua aplicação na prática clínica.

Com o nascimento de um RNPT, faz-se necessário um local que possua recursos tecnológicos, humanos e terapêuticos especializados a fim de proporcioná-lo cuidados mais complexos. Esses suportes são encontrados nas UTIN, onde a assistência ocorre de maneira contínua, e cujo surgimento tem proporcionando um aumento na sobrevida orgânica dos prematuros, principalmente os extremos e de baixo peso.

Entretanto, a hospitalização do RNPT na UTIN está associada à sua submissão a um número excessivo de procedimentos como as punções venosas, as sondagens orogástricas e vesicais, as glicemias capilares, a realização de curativos, a aspiração de vias aéreas e a entubação endotraqueal dentre outros, o que pode gerar desconforto, estresse e dor.

Um recém-nascido prematuro na UTIN recebe cerca de 130 a 234 manipulações nas 24 horas, sendo que muitas dessas manipulações são dolorosas. Além disso, ao ser internado em uma UTIN, o RNPT está entrando num ambiente totalmente diferente do útero materno ${ }^{(2)}$. Os ruídos sonoros são altos e as luzes fortes e contínuas; a ação da gravidade impede seus movimentos e passa a ser excessiva, além de ser imprevisivelmente manuseado, muitas vezes, sem o cuidado adequado para diminuição do estresse e da dor ${ }^{(3)}$.

Nesta direção, é preciso considerar que o RNPT é capaz de sentir dor e que a tradicional cultura de que a falta de mielinização seja um indicador de imaturidade do sistema nervoso central, para apoiar o argumento de que este não é capaz de sentir o processo doloroso, necessita ser desconstruído, a fim de se implementar cuidados para aliviar este desconforto.

Por conseguinte, diante do grande número de RNPT que são submetidos a diversos eventos estressantes ou dolorosos e ao considerar a subjetividade da dor e a inabilidade em relatá-la verbalmente, os enfermeiros das unidades neonatais devem estar atentos a essa linguagem tão peculiar, expressa através de alterações comportamentais e fisiológicas promovendo, assim, o cuidado integral e seguro ao prematuro ${ }^{(4)}$.

Na prevenção e controle da dor e do estresse no RNPT “os profissionais de saúde devem fazer uso de intervenções ambientais não farmacológicas e farmacológicas apropriadas para prevenir, reduzir ou eliminar o estresse e a dor nos neonatos" $^{\prime(5)}$.

Sendo assim, considera-se imperativo a avaliação e a intervenção no processo doloroso na população de prematuros, tendo em vista o potencial para alterações no seu desenvolvimento neuropsicomotor. Desta forma, este processo precisa ser uma constante na prática clínica dos trabalhadores da saúde em cuidados intensivos, para que a atenção ao RNPT possa ser ofertada com excelência, segurança e humanização. Neste contexto, este estudo tem como objeto a avaliação e intervenção no processo doloroso em RNPT realizadas pela equipe de enfermagem de um hospital público da Bahia.

O interesse por este estudo surgiu na prática diária no cuidado ao RNPT, ao observar empiricamente que a equipe de enfermagem da UTIN do hospital geral de uma cidade da Bahia realizava diversos procedimentos invasivos sem oferecer suporte não farmacológico ou farmacológico para o alívio ou prevenção do fenômeno da dor. Ademais, nesta unidade, a equipe de enfermagem registrava a cada três horas os sinais vitais dos recém-nascidos internados, não considerando a dor como o quinto sinal a ser avaliado.

Isto posto, questionou-se: Como a equipe de enfermagem de uma UTIN avalia o processo doloroso em RNPT? Como esta equipe alivia este fenômeno? Desta maneira, este estudo tem como objetivos os de analisar os parâmetros utilizados pela equipe de enfermagem de um hospital público da Bahia para a avaliação da dor no RNPT; e descrever as intervenções utilizadas pela equipe de enfermagem para aliviar a dor no RNPT.

\section{MÉTODOS}

Este estudo foi do tipo descritivo e de natureza qualitativa, a qual permitiu avaliar a compreensão da experiência humana como ela é vivida, coletando e analisando materiais narrativos e subjetivos ${ }^{(6)}$.

O campo empírico foi um hospital público de Feira de Santana, no estado da Bahia. Hospital de médio porte que atende mulheres procedentes de todo o município e regiões circunvizinhas e cujas unidades neonatais possuem 16 leitos no total e contam com uma equipe multiprofissional. A UTIN conta com seis médicos neonatologistas, uma enfermeira gerente, dez enfermeiras neonatais, vinte profissionais de enfermagem de nível médio e duas técnicas administrativas.

Para a escolha destes participantes foram utilizados os seguintes critérios de inclusão: Ser funcionário da UTIN; Ter tempo de atuação na unidade superior a um ano; Ser funcionário assistencial; Estar escalado para cuidar de RNPT com necessidade de procedimentos invasivos; Aceitar participar do estudo mediante leitura e assinatura do Termo de Consentimento Livre e Esclarecido. Foram excluídos os funcionários que estavam de férias ou licença e os que prestavam ações burocráticas ou administrativas. Sendo assim, participaram deste estudo dez profissionais da equipe de enfermagem da UTIN em estudo. Este número de entrevistadas deu-se mediante a saturação teórica dos dados.

Para a garantia de que os direitos e o bem estar dos participantes da pesquisa fossem respeitados, atendeu-se às exigências 
da Resolução 196/96 do Conselho Nacional de Saúde( ${ }^{(7)}$, que recomenda a observação dos princípios éticos na pesquisa. Assim, este estudo foi aprovado pelo Comitê de Ética na Pesquisa da Faculdade de tecnologia e Ciências, campus de Salvador-BA, sob o Parecer No 0574-2008. Todas as entrevistadas selecionadas leram e assinaram o Termo de Consentimento Livre e Esclarecido. Ademais, cada uma delas foi identificada com códigos conforme a ordem de realização das entrevistas, para preservar a identidade das mesmas.

Os dados foram coletados no período dezembro de 2008 a fevereiro de 2009, através de entrevistas semiestruturadas, sendo utilizado um roteiro que continha informações relativas às entrevistadas e as seguintes questões norteadoras: Como você percebe que um recém-nascido prematuro está sentindo dor durante a realização de um procedimento invasivo? Quais intervenções você utiliza para amenizar a dor no RNPT internado nessa unidade neonatal durante estes procedimentos?

As entrevistas foram transcritas na íntegra, sendo analisadas por meio da Análise de Conteúdo de Bardin. Inicialmente realizamos uma leitura flutuante das entrevistas transcritas, com o intuito de conhecermos a natureza do conteúdo das mesmas. Em seguida, foi feita uma leitura mais aprofundada a fim de descobrirmos o conteúdo latente que se encontrava nos discursos coletados, sendo identificadas duas categorias analíticas, a saber: Reconhecendo a dor através de sinais do recém-nascido e As intervenções da equipe de enfermagem.

\section{RESULTADOS E DISCUSSÕES}

Neste estudo foram entrevistadas dez participantes, que faziam parte da equipe de enfermagem da UTIN do hospital em estudo, assim distribuídas: cinco enfermeiras e cinco técnicas de enfermagem. Na tabela 1 é possível visualizar o perfil das dez entrevistadas.

Todas as participantes deste estudo eram do sexo feminino. A idade das entrevistadas variou de 25 a 47 anos. O tempo de atuação na UTIN foi de 01 ano e 05 meses a 14 anos.

\section{RECONHECENDO A DOR ATRAVÉS DE SINAIS DO RECÉM-NASCIDO}

Apesar de reconhecerem a importância de avaliação da dor nos recém-nascidos prematuros internados na UTIN, a equipe de enfermagem ainda não utiliza escalas para a avaliação deste processo, bem como não há uma política setorial, que vislumbre a dor como um dos parâmetros vitais a serem avaliados segundo o protocolo do serviço.

Nós não utilizamos nenhuma escala de dor neste setor. Não temos treinamento sobre este assunto. (E1)

Não utilizo nenhum tipo de escala para saber se o prematurinho está sentido dor. (E5)

Eu faço a avaliação conforme sinais do recém-nascido. Eu não uso nenhuma escala, pois aqui não tem implantado este instrumento. (E6)

Eu acredito que o recém-nascido sente a dor. Só que eu aqui não tem escalas impressas no prontuário para ser utilizadas por nós. Eu acho que ele (recém-nascido) está sentido dor quando chora e fica agitado. (E9)

Tabela 1 - Caracterização das entrevistadas. Feira de Santana-BA, 2009.

\begin{tabular}{|c|c|c|c|c|c|}
\hline Código & Categoria & Sexo & Idade (anos) & Formação & Atuação na UTIN \\
\hline E1 & Enfermeira & Feminino & 28 & 06 anos & 05 anos \\
\hline E2 & Enfermeira & Feminino & 33 & 07 anos & 05 anos \\
\hline E3 & Enfermeira & Feminino & 25 & 01 ano & 01 ano e 5 meses \\
\hline E4 & Enfermeira & Feminino & 41 & 09 anos & 07 anos \\
\hline E5 & Enfermeira & Feminino & 37 & 08 anos & 06 anos \\
\hline E6 & TE* & Feminino & 29 & 03 anos & 02 anos e 10 meses \\
\hline E7 & TE & Feminino & 27 & 10 anos & 5 anos \\
\hline E8 & TE & Feminino & 34 & 10 anos e 4 meses & 8 anos \\
\hline E9 & TE & Feminino & 47 & 15 anos e dois meses & 10 anos \\
\hline E10 & $\mathrm{TE}$ & Feminino & 45 & 16 anos & 14 anos \\
\hline
\end{tabular}

*TE - Técnica de Enfermagem da UTIN. 
As escalas de dor são instrumentos que facilitam a interação e comunicação entre os membros da equipe de saúde, permitindo avaliar a evolução da dor em cada paciente e a verificar a resposta frente à terapia analgésica ${ }^{(8)}$. Sendo assim, a avaliação implementada por esta equipe, em relação ao reconhecimento do processo doloroso no RNPT, realiza-se de forma empírica, não sistematizada e sem evidências científicas, pautada apenas em um dos parâmetros do contexto global das escalas de avaliação da dor. Essa avaliação precisa ser incorporada na prática destes profissionais de saúde como outro sinal vital e que necessita receber a devida atenção, com vistas à redução do impacto provocado pelo fenômeno da dor no processo de crescimento e desenvolvimento do RNPT.

Assim, conforme análise das falas das entrevistadas evidenciou-se que, no contexto de sua prática clínica na UTIN, a equipe de enfermagem reconhece a dor por meio da avaliação do choro e de manifestações do recém-nascido, através de sua expressão facial.

Através do choro, das faces de dor que ele apresenta, a gente observa o franzir da testa, o choro muito alto e por aí vai. (E1)

Choro, agitação e características de sua expressão facial. (E3)

O choro é um dos sinais e o outro sinal é estar irritado. (E5)

[...] Eu acho que ele (recém-nascido) está sentido dor quando chora e fica agitado. (E9)

O choro foi um dos sinais mais apontados como parâmetro clínico de avaliação do processo doloroso no RNPT, pois o mesmo é uma forma de comunicação e manifestação deste. Esta variável, apesar de ser um indicativo de que algo errado acontece, pode ou não estar relacionado à dor. Nessa situação, possui uma fase expiratória mais prolongada e tem durabilidade aumentada, sendo de difícil controle ${ }^{(4)}$.

É necessário que a equipe de enfermagem fique bastante atenta em relação à qualidade do choro apresentado por um recém-nascido, já que nem sempre esse pode ser considerado um indicador clínico de dor. Na maioria das vezes, pode ocorrer devido a estímulos tais como a fome, a angústia, as cólicas abdominais, a agitação, o sono, à presença de dispositivos do cuidado neonatal, como a sonda orogástrica e vesical, e o desconforto. O choro é pouco específico, pois pode ser desencadeado por outros estímulos não dolorosos. Assim, o choro isoladamente é uma medida pouco efetiva na avaliação da dor ${ }^{(9)}$.

Desta maneira, ao se realizar uma avaliação clínica de parâmetros sugestivos do processo doloroso no RNPT, faz-se necessário a verificação do ambiente ao redor do bebê, bem como a avaliação mais acurada da intensidade e do estado de saúde do mesmo.

Diferente da criança e do adolescente, a dor no recém-nascido se manifesta de forma não verbal através de alterações na dinâmica fisiológicas e de seu comportamento. As entrevistadas reconhecem que o RNPT sente dor através de manifestações comportamentais e variáveis fisiológicas.
[...] Expressão facial, agitação psicomotora, taquicardia e alterações na saturação de oxigênio. (E2)

Eu observo se ele apresenta choro, sua expressão facial, que pode demonstrar a testa franzida, os olhos fechados, a taquipnéia e a taquicardia. (E4)

[...] Expressão facial, na agitação do bebê, taquipnéia, hiperatividade [...]. (E6)

[...] Através da expressão facial, choro, agitação, taquicardia, taquipnéia, quedas na saturação de oxigênio no monitor cardíaco e dificuldades para dormir. (E7)

A equipe de enfermagem avalia a dor, principalmente, observando mudanças no comportamento da criança, incluindo choro característico, alterações na mímica facial, no humor e nos movimentos corporais ${ }^{(10)}$.

Cada profissional da equipe de enfermagem percebe a dor conforme vivência profissional e científica, bem como pela influência cultural. Em suas manifestações, a dor é percebida mediante alterações comportamentais e fisiológicas dos recém-nascidos. Dentre as alterações comportamentais destacaram o choro, expressão facial, resposta motora, irritabilidade e alterações de sinais vitais ${ }^{(8,11)}$.

Além disso, o fato de a dor ser um fenômeno subjetivo gera uma grande dificuldade para a elaboração de um método único de avaliação e de fácil aplicação na prática clínica dos profissionais das unidades neonatais ${ }^{(12)}$.

As medidas fisiológicas são usadas para o reconhecimento da algia no neonato. Dentre elas podemos citar o aumento da freqüência respiratória, da freqüência cardíaca, da saturação do oxigênio, da pressão arterial, da pressão intracraniana, a sudorese palmar e o tônus vagal. Em relação aos parâmetros comportamentais, o RNPT pode apresentar movimentos corporais, o choro característico, a expressão facial, o padrão de sono e vigília. Estes dados foram semelhantes aos resultados de outros estudos nacionais ${ }^{(8,11,13-14)}$.

Estímulos dolorosos agudos desencadeiam nos recém-nascidos uma resposta global ao estresse, que inclui modificação a nível cardiovascular, respiratório, imunológico, hormonal e comportamental, entre outros. Essas respostas fisiológicas são acompanhadas de uma reação endócrino-metabólica de estresse, com liberação, entre outros hormônios, de adrenalina, noradrenalina e cortisol, podendo também resultar em hiperglicemia e catabolismo protéico lipídico, interferindo no equilíbrio homeostático, que no $\mathrm{RN}$ já é precário ${ }^{(15)}$.

Além disso, algumas doenças, bem como medicamentos sedativos e analgésicos podem interferir nas respostas comportamentais de dor. $\mathrm{O}$ uso de drogas vasoativas também pode promover alterações fisiológicas semelhantes às resultantes de dor. Outro fator limitante para o uso de indicadores comportamentais é a presença de cânula traqueal que dificulta constatar a ocorrência de choro e mímica facial ${ }^{(13)}$.

O grande desafio na avaliação da dor consiste em compreender a diferença do que é dor ou desconforto, para estabelecer assim, um correto diagnóstico deste processo. Esta 
é uma das principais dificuldades da equipe de enfermagem, geralmente, devido a subjetividade da dor e pela falta de treinamento dos profissionais ${ }^{(14)}$.

Desta maneira, ao avaliar a ocorrência da dor no RNPT utilizando parâmetros pontuais isolados do contexto global da internação do recém-nascido na UTIN, esta equipe de enfermagem poderá implementar medidas ineficazes no manejo clinico deste fenômeno. Por outro lado, a ausência da aplicação das escalas de avaliação da dor pode implicar na maior dificuldade de identificação da dor no RNPT, retardando a implementação de cuidados efetivos e potencializando alterações na sua condição orgânica.

\section{AS INTERVENÇÕES DA EQUIPE DE ENFERMAGEM}

Para prestar o cuidado ao recém-nascido com dor é importante que o profissional esteja atento a sua comunicação não verbal, mas sim a linguagem corporal. Quando se tem o relacionamento afetivo, a percepção da necessidade do cuidado pode ser mais facilmente percebida. Ao compartilhar os momentos de emoções, sofrimento e, até mesmo de vitória, saindo da visão tecnicista moderna, o profissional pode refletir se o sucesso terapêutico não poderia ter sido alcançado através, ou com a ajuda, dessa atenção, da sensibilidade e dos sofrimentos demonstrados durante o cuidado ${ }^{(4)}$. Na análise da fala das entrevistadas percebeu-se que a equipe de enfermagem promove $o$ conforto do recém-nascido como forma de alívio da dor.

Normalmente, nós utilizamos as intervenções não farmacológicas: contenção, diminuição do barulho, de luz, excesso de luz, o toque no recém-nascido, tudo isso conforta mais o bebê no momento de um procedimento invasivo. (E1)

[...] Faz uma chupetinha com o látex da luva e aí coloca as gotinhas, aí ele vai sugar, vai sentir prazer sugando e aí vai amenizar a dor [...] muda de decúbito, fazer massagem de conforto [....]. (E2)

Nas falas destas entrevistadas, nota-se que há uma preocupação com o conforto e o bem-estar no prematuro, sendo implementadas algumas medidas, tais como a contenção, a diminuição do barulho e do excesso de luz da UTIN, a mudança de decúbito, o toque e a sucção não nutritiva. Estes dados foram encontrados em dois estudos realizados no Brasil ${ }^{(11,14)}$.

Diante da intensidade da luz nas Unidades Neonatais, as ações de enfermagem devem ser dirigidas no sentido de reduzir a luminosidade ambiental, promovendo estratégias tais como, cobrir a incubadora com mantas, evitando desta forma, o excesso de estímulos, além de proporcionar o ciclo natural de sono e vigília do RNPT ${ }^{(16)}$. O toque e o contato físico também acalmam. A estimulação sensorial poderá ser útil como, por exemplo, o uso da música, a fala suave, as massagens e o estímulo visual(4).

Outra forma de acalmar o RNPT é a contenção do recém-nascido em um ninho, denominado útero artificial, o qual pode ser improvisado para promover a sua organização comportamental( ${ }^{(4)}$.

[...] Utiliza as intervenções não farmacológica, são aquelas: contenção [...] tudo isso conforta mais o bebê no momento de um procedimento invasivo. (E3)

Na mudança de decúbito, alimentação, sucção, procura de todas as formas manter confortável até que identifique a causa. (E4)

[...] Eu normalmente coloco o prematuro enroladinho, para contê-lo durante a punção venosa periférica, trabalho em dupla e utilizo a chupetinha com glicose. (E8)

[...] Para aliviar a dor quando eu acho que o bebê prematuro está sentindo, eu normalmente procuro mantê-lo organizadinho através do conforto no útero artificial que nós fazemos todos os dias com rolinhos aqui da unidade, além de acalmá-lo, colocar a luvinha na boca para ele sugar e falo com a enfermeira que vou dar um pouquinho de glicose a $25 \%$. (E10)

A equipe de enfermagem pode e deve utilizar as medidas não farmacológicas de forma constante para o controle da dor nas unidades neonatais, já que são estratégias de cuidado que não necessitam da interferência de outros profissionais. As medidas de conforto e controle da dor como o toque, o contato pele-pele, a nutrição não nutritiva, dentre outras, podem ser realizados sistematicamente com vistas a prevenir a dor, limitá-la e restabelecer o bem-estar do recém-nascido.

A literatura relata que os benefícios da estimulação da sucção não nutritiva variam desde a adequação da musculatura oral, da regulação dos estados de consciência do bebê, do ganho de peso, recebendo a mesma quantidade calórica, da alta precoce, da facilidade de digestão, da transição para a alimentação por via oral mais rápida e mais fácil, dentre outras. Entretanto, as indicações ou parâmetros para início de uma sucção não nutritiva não são unânimes, não havendo indicadores precisos sobre quando iniciá-lo, como deve ser e ainda como proceder para levar o recém-nascido à prontidão para a alimentação oral|(17).

A sucção não nutritiva e a solução glicosada utilizadas como medidas não farmacológicas, para o alívio da dor no recém-nascido prematuro, amenizam a dor no recém-nascido durante a realização de procedimentos. O emprego da sucção não nutritiva através da chupeta em unidades neonatais está indicado nos procedimentos dolorosos, pois inibe a hiperatividade e modula o desconforto, ajudando na organização neurológica e emocional do neonato, diminuindo a duração do choro, acalmando-o mais rapidamente, além de reduzir as frequências cardíacas e respiratórias ${ }^{(4)}$.

Nos discursos das entrevistadas ficou evidente que as mesmas utilizam da solução glicosada nos diversos contextos de sua prática diária na unidade neonatal para amenizar o processo doloroso no RNPT:

Eu faço a prevenção dessa dor utilizando a glicose, os bebês a termo a gente utiliza $1 \mathrm{ml}$ de glicose antes do procedimento doloroso e no caso da glicose vai estimular a serotonina e o que vai dar o prazer ao bebê então vai amenizar essa dor, é uma prevenção que a gente faz. (E1) 
[...] E quando o bebê prematuro, a gente coloca uma gotinha de glicose antes do procedimento e faz uma chupetinha com o látex da luva e ai coloca as gotinhas ai ele vai sugar, vai sentir prazer sugando e ai vai amenizar a dor. (E2)

[...] A fisioterapeuta ensinou a gente a fazer o bico com a luva e botar glicose a $25 \%$, três gotinhas. Geralmente isto acalma o bebê e a gente faz o procedimento. (E3)

Procuro acalmar deixando confortável, coloco um bico artificial e utilizo glicose. (E5)

Nós utilizamos muito aqui a sucção não nutritiva com chupetinha de luva e glicose [...] (E6)

Como pode ser visto que a equipe de enfermagem do hospital em estudo reconhece e coloca em prática o uso da sucção-nutritiva e a glicose com o objetivo de amenizar a dor no RNPT. Entretanto, nota-se que este cuidado acontece de forma não sistematizada e indiscriminada, já que nesta UTIN não foi encontrado protocolo assistencial de enfermagem que abordasse esta conduta no manejo clínico da dor no prematuro.

A utilização da glicose, antes do procedimento doloroso, irá reduzir a duração do choro, da freqüência cardíaca e a expressão facial ${ }^{(18)}$. Por conseguinte, a sucção não nutritiva é um ponto positivo e pode ser utilizado como auxílio na terapêutica, pois foi comprovado que o mesmo trás benefícios a saúde e a recuperação do neonato.

Apesar de a Academia Americana de Pediatria e a Sociedade Pediátrica Canadense recomendarem o uso da solução de sacarose como rotina para alívio da dor em UTIN durante procedimentos invasivos e dolorosos, os estudos sobre o uso da substância em doses repetidas ainda não são conclusivos e merecem novas investigações. Nestas, a avaliação da eficácia da administração em doses repetidas da sacarose no alívio de dor em neonatos deve necessariamente ampliar o estudo dos possíveis efeitos colaterais, tanto do ponto de vista da saúde quanto do desenvolvimento, assim como controlar o número de procedimentos invasivos ${ }^{(19)}$.

Nota-se uma constante preocupação desta equipe de enfermagem na promoção de um cuidado individualizado ao RNPT no controle de sua dor, através da implementação baseada em evidências científicas. Entretanto, mesmo com a aplicação das estratégias não farmacológicas, é fundamental o investimento em educação permanente nesta instituição, com vistas à capacitação desta equipe, para que o processo doloroso possa ser considerado na avaliação global do prematuro como o quinto sinal vital.

\section{CONSIDERAÇÕES FINAIS}

Percebeu-se que a equipe de enfermagem identifica a dor no recém-nascido prematuro de maneira não sistematizada e fragmentada. E ainda, não existe nenhum tipo de impresso ou escala para auxiliá-las na identificação mais precisa do processo doloroso. As escalas funcionam como um ponto chave tanto para a identificação do processo doloroso, bem como para a adequação do tratamento.

Com relação ao manejo da dor no RNPT pela equipe de enfermagem, observou-se que as condutas adotadas são condizentes com a literatura. Esses profissionais conhecem os métodos alternativos e sua importância e sabem identificar um melhor método para melhorar a qualidade de vida do RNPT minimizando assim, as sensações dolorosas.

Entretanto, em virtude do fato de as escalas não fazerem parte do contexto de sua prática clínica diária, como ferramenta do cuidado para a avaliação da dor no neonato prematuro, estas intervenções podem se configurar em iatrogenias, potencialmente capazes de provocar danos ao recém-nascido, infringindo os princípios da segurança do paciente.

A equipe de enfermagem deverá levar em consideração os aspectos inerentes ao processo doloroso, bem como considerá-lo como um indicador potencial de alterações na sobrevida orgânica do recém-nascido prematuro, tendo em vista as suas repercussões nos sinais vitais e comportamento do mesmo.

Sugere-se a elaboração e implementação de protocolos assistenciais nesta unidade, bem como a inclusão da avaliação do processo doloroso como sinal vital de suma importância, a ser avaliado no conjunto da aferição dos outros dados vitais, por meio de instrumentos do cuidado, tais como as escalas neonatais, com vistas à padronização da identificação e como guia clínico para o tratamento efetivo e imediato do mesmo.

Por outro lado, faz-se mister a inclusão da dor como sinal de adaptação inadequada do prematuro ao espaço da Unidade Neonatal, bem a mudança de filosofia institucional, com vistas à valorização do tratamento deste sinal como um indicador de qualidade da atenção dispensada ao RNPT.

Desta maneira, este estudo poderá subsidiar a equipe de enfermagem na organização de seu processo de trabalho, com o intuito e proporcionar uma passagem tranqüila do neonato prematuro pela unidade neonatal, com vistas à importância do cuidado de excelência, de qualidade e humanizado.

\section{REFERÊNCIAS}

1. Araújo DMR, Pereira N de L, Kac G. Ansiedade na gestação, prematuridade e baixo peso ao nascer: uma revisão sistemática da literatura. Cad de Saúde Pública 2007;23(4):747-56.

2. Costa H de PF, Tadeu S. O Recém - Nascido de Muito Baixo Peso. São Paulo: Atheneu; 2004.
3. Ministério da Saúde (Brasil) Secretaria de Políticas de Saúde, Área de saúde da criança. Atenção humanizada ao recém-nascido pré-termo de baixo peso: Método Mãe Canguru - Manual do curso. Brasília: Ministério da Saúde; 2002.

4. Calasans, MT de A. A dor no recém-nascido no cotidiano da Unidade de Terapia Intensiva Neonatal. Salvador. 
Dissertação [Mestrado em Enfermagem]- Universidade Federal da Bahia; 2006.

5. Gaíva MAM, Dias $\mathrm{N}$ da S. Dor no recém-nascido: percepção de profissionais de saúde de um hospital universitário. Rev Paul Enferm 2002;21(3):234-9.

6. Polit DF, Hungler BP. Fundamentos de pesquisa em Enfermagem: métodos, avaliação e utilização. 5. ed. Porto Alegre: Artmed; 2004.

7. Ministério da Saúde (Brasil) Conselho Nacional de Saúde, Comissão Nacional de Ética em Pesquisa. Resolução $n^{\circ}$ 196 de 10 de outubro de 1996. Diretrizes e normas regulamentadoras de pesquisa envolvendo seres humanos. Diário Oficial da União 10 out 1996.

8. Crescêncio EP, Zanelato S, Leventhal, LC. Avaliação e alívio da dor no recém-nascido. Rev Eletrônica Enferm [periódico na internet]. 2009 [acesso em: 19 out 2010];11(1) Disponível em: http://www.fen.ufg.br/revista/v11/ $\mathrm{n} 1 / \mathrm{v} 11 \mathrm{n} 1 \mathrm{a} 08 . \mathrm{htm}$.

9. Couto P. Dor sem palavras: Reações similares às mais variadas sensações trazem confusão na hora de detectar problemas nos bebês. Rev Hebron 2006;23:20-2.

10. Oliveira RM, Silva AVS, Chaves EMC, Sales NC. Avaliação comportamental e fisiológica da dor em recém-nascidos pelos profissionais de enfermagem. REME - Rev Min Enferm 2010; 14(1):19-24.

11. Veronez $M$, Corrêa DAM. A dor e o recém-nascido de risco: percepção dos profissionais de enfermagem. Cogitare Enferm 2010;15(2):263-70.
12. Balda RCX, Guinsburg R. Avaliação da dor no período neonatal. In: Kopelman BI, Santos AMN, Goulart AL, Almeida MFB, Miyoshi MH, Guinsburg R. Diagnóstico e tratamento em neonatologia. São Paulo: Atheneu; 2004.

13. Bueno M, Kimura, AF, Pimenta CAM. Avaliação da dor em recém-nascidos submetidos à cirurgia cardíaca. Acta Paul Enferm 2007;20(4):428-33.

14. Scochi, CGS, Carletti M, Nunes R, Furtado MCC, Leite AM. A dor na Unidade Neonatal sob a perspectiva dos profissionais de enfermagem de um hospital de Ribeirão Preto-SP. Rev Bras Enferm 2006;59(2):188-94.

15. Guinsburg R. Dor no recém-nascido: importância do estudo da dor no recém-nascido. In: Rugolo LMS. Manual de neonatologia. Rio de Janeiro: Revinter; 2000. p.63-9.

16. Scochi CGS, Riul MJS, Garcia CFD, Barradas L da S, Pileggi $S$ de O. Cuidado Individualizado ao pequeno prematuro: o ambiente sensorial em unidade de terapia intensiva neonatal. Acta Paul Enferm 2001;14(1):9-16.

17. Caetano, LC, Fujinaga, Cl, Scochi CGS. Sucção não-nutritiva em bebês prematuros: estudo bibliográfico. Rev Latinoam Enferm 2003;11(2):232-6.

18. Gaspary LV, Rocha I. Intervenções não-farmacológicas para o alivio da dor em Recém-nascidos Prematuros (RNPT). Rev Nurs 2004;79(7):47-50.

19. Gaspardo CM, Linhares MBM, Martinez FE. A eficácia da sacarose no alívio de dor em neonatos: revisão sistemática da literatura. J Pediatr 2005;81(6):435-42. 